\title{
Metadato de la Colección Biológica de Corales (Escleractinia) del Museo Javeriano de Historia Natural Lorenzo Uribe S.J.
}

\author{
Martha Y. Vallejo' y Alberto Acosta PhD² \\ 1,2Pontificia Universidad Javeriana, Facultad de Ciencias, Departamento de Biología. Unidad de Ecología y Sistemática (UNESIS).

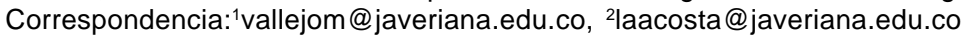 \\ Recibido: 27-04-05 / Aceptado: 27-08-05
}

\begin{abstract}
Resumen
Día a día surge la necesidad de divulgar a la comunidad científica nacional e internacional la información existente en las colecciones biológicas de los museos, particularmente cuando son pocas para un taxa en el país, o cuando es difícil acceder a ellas. Aunque las bases de datos en general compilan información de forma sistemática, las pertenecientes a las colecciones biológicas del Museo Javeriano de Historia Natural Lorenzo Uribe S.J. (MUJ) aún no están estandarizadas de manera tal que puedan ser divulgadas para una consulta universal. Para solucionar este problema en el MUJ se ha hecho uso de herramientas como los metadatos, datos sobre datos, que a partir de la base de datos de la colección de corales resume información de su contenido, calidad, donde fue colectado y condición; brindando acceso a información en un lenguaje homogéneo. Los pasos seguidos para generar el metadato de la colección de corales referenciados en el MUJ fueron: revisión, digitación, organización, depuración y estandarización a nivel taxonómico y geográfico de la información de cada uno de los datos de los registros biológicos, tomando como base estándares internacionales. El metadato condensó información sobre: 1. Géneros de Scleractinios del MUJ, 2. Citación del autor del metadato y del listado de Scleractinios; 3. Cobertura geográfica de colecta; 4. Condiciones de acceso y uso; y 5. Información temporal e hipervínculos, entre otros aspectos. Así mismo se aplicó el índice de biodiversidad de metadatos para cualificar interoperabilidad con otros metadatos y su divulgación. El metadato de corales del MUJ es eficaz (76.6\%) porque facilita la búsqueda eficiente, específica y completa de la información sobre corales en el Caribe colombiano, ya que solo existen dos colecciones referenciadas en el país. Además, da a conocer parte del patrimonio y biodiversidad y permite comparar e intercambiar información existente a nivel internacional vía electrónica.
\end{abstract}

Palabras claves: corales, metadato, Museo Javeriano, escleractinios.

\begin{abstract}
Coral Collection Metadata (Scleractinia) from the Museo de Historia Natural Lorenzo Uribe S.J.: The necessity to notify scientist about the biological collections is a daily need, particularly when there are few of them in a country for a particular taxa, or when it's difficult the access to them. Although the collection data bases compile information in systematic form, they are not design to divulgate the information in a universal language. To solve this problem, tools such as the metadata were created, data over data, they use the collection
\end{abstract}


data base to summary the information contained, their quality; where they were collected, and state; allowing information access in a universal language. The steps we followed to generate the metadata from the coral collection referenced to the Museo de Historia Natural Lorenzo Uribe S.J. (MUJ) were: revision, digitations, organization, depuration, and standardization at taxonomic and geographic level for each biological specimen, using an international standard. The metadata allow us to know the Scleractinian genera list (MUJ), and to condense information on: 1. author and Scleractinian list citations; 2. Geographic sampling cover; 3. Conditions to access and use; and 4. temporal information and hipervinculum; within other aspects. In the same way, we applied the biological index of metadata documentation (76.6\%) to quantify inter-operability with other metadata and it divulgation. Coral MUJ metadata is effective because it facilitate search of coral information in Colombia, in an efficient, specific, and complete way, this is because there are only two collections referenced. In addition it will allow us to show our biodiversity and to compare and interchange the information internationally.

Key word: corals, metadata, Museo Javeriano, scleractinia.

\section{Introducción}

Aunque el término metadato es relativamente nuevo, lo que representa como tal no lo es, desde los años 60 con la intención de facilitar el trabajo a los investigadores y usuarios de las bibliotecas internacionales se empezaron a compartir metadatos descriptivos usando sistemas automatizados como catálogos de acceso público y reglas de catalogación internacionalmente aceptadas para con ello lograr integrar, facilitar y divulgar información (1).

Desde entonces se ha venido utilizando el término literalmente con un significado de "datos sobre datos" (2) que brindan información resumida, organizada y estandariza de datos existentes utilizados para extraer la información relevante desde cualquier objeto, entidad o recurso de manera rápida y eficaz (3). Para las colecciones biológicas de los museos del mundo, el metadato ofrece una herramienta cuantitativa y cualitativa de los ejemplares presentes en cada colección. El metadato sintetiza información taxonómica, geográfica de cantidad y calidad de los datos (4). Así mismo, el material impreso, audiovisual o geoespacial son otros ejemplos a los que se les puede extraer información para generar el metadato (5).

El metadato de una colección biológica sirve para: (i) buscar información rápida sobre cada grupo especifico o colección; (ii) conocer información de referencia; (iii) evitar re-analizar la información

existente; (iv) conocer la fecha próxima de revisión y actualización; (v) presentar información de la versión del estándar utilizado; (vi) conocer la fecha de creación de la colección; y (vii) encontrar los listados o publicaciones existentes de diferentes taxa (6).

Pese a la diversidad existente en Colombia, existen pocos metadatos de colecciones biológicas, entre los cuales se destacan los del Instituto de Recursos Biológicos Alexander von Humboldt (IAvH) para grupos como Hymenoptera, Lepidoptera (7); y los metadatos de las colecciones del Museo de Historia Natural Marina de Colombia (MHNMC), los cuales son publicados en el Sistema de información sobre biodiversidad (SIB) y en el Sistema de Información sobre Biodiversidad Marina (SIBM), respectivamente (7). La elaboración y divulgación de metadatos de colecciones biológicas en Colombia, es un proceso reciente, 2002, que requiere ser incentivado en las diversas instituciones (7). Actualmente la Comunidad Europea, Chile y México dentro del marco de la biodiversidad, están implementando el uso de los metadatos para cada grupo biológico y/o colecciones, y así dar a conocer de manera general y eficiente la información que representa el patrimonio de su país (5).

En Colombia aunque parte de la biodiversidad esta representada en colecciones de diferentes museos, la mayoría de esta información no esta disponible ni se intercambia entre museos por falta de estandarización 
de las bases de datos. Para homogenizar y estandarizar la información de los registros biológicos de las colecciones existen estándares internacionales, Dublin Core (8), que definen campos claves y casillas para completar y de uso obligatorio. Cualquier colección biológica que utilice este estándar podrá intercambiar información con otras que lo utilicen (9). La información que se describe en un metadato debe ser completa y precisa respecto a los campos obligatorios definidos por la institución; veraz y sin inferir, extrapolar, deducir, o modificar datos (9). Para determinar la pertinencia de publicar la información de un metadato se hace necesario aplicar herramientas metódologicas e índices de metadatos a partir de la información documentada, y dependiendo de las prioridades de cada museo se analizan los resultados bajo criterios cuantitativos y cualitativos. La información contenida en el metadato de la colección de escleractinios se hace relevante a nivel nacional y mundial debido a que hace parte de la colección de invertebrados marinos del MUJ, y por que facilita que la compilación de información de especies preservadas en las colecciones biológicas constituyan un testimonio de la biodiversidad pasada y presente de un país. En este artículo se realza la importancia de generar el metadato de una colección biológica y se presenta el estándar de conjunto de datos utilizado, con cada uno de sus campos obligatorios debidamente diligenciados a partir de la base de datos de corales del MUJ, que previamente se ha estandarizado en un formato para registros biológicos. Para organizar la información anexa a los registros biológicos del MUJ, fue necesario consultar los formatos ya establecidos de metadatos, como: Graphic Representation NBII Content of Standard (10), NBII Content of Standard for National Biological Information Infraestructure Metadata (11), FDGC Content of Standard for Digital Geospatial Metadata (12) y Dublín Core (8).

\section{Materiales y Métodos}

El metadato se generó usando la base de datos de la colección de corales Escleractinios del MUJ com- puesta por la información de campo anexa a cada uno de los ejemplares. Cada uno de los datos de los registros biológicos se sistematizó en excel y se les asignó un número de referencia a cada registro biológico, dentro de la colección de invertebrados marinos. Los criterios tenidos en cuenta para ingresar los datos de los registros biológicos a la base de datos fueron ejemplares: (i) en buen estado; (ii) con datos mínimos obligatorios: acrónimo, numero, fecha de colecta, distribución geográfica, rango batimétrico, coordenadas geográficas; datos sin ambigüedad (612); (iii) determinados taxonómicamente, mínimo a familia. Aquellos ejemplares que no cumplían con estos criterios no se incluyeron a la base de datos y se les destinó como material de docencia en la Pontificia Universidad Javeriana (PUJ). Una vez creada y alimentada la base de datos, la información de los registros biológicos se estandarizó en un formato siguiendo a Rivera (6).

Este procedimiento consistió en organizar la información anexa a los ejemplares a nivel taxonómico y geográfico por jerarquía, regiones, sitios, biomas, departamentos, país etc., en cada una de las casillas correspondientes al formato para registros biológicos (6). Una vez se le asignó una casilla a cada dato de cada registro biológico y se realizó la estandarización, se generó el metadato de la colección biológica de escleractinios, utilizando el estándar para la documentación de conjunto de datos (9). Posteriormente, se aplicó el indicador de intercambio de información; hoja metodológica realizada por el IAvH (datos no publicados) llamado índice de documentación para metadatos y su fórmula es: $\mathrm{PM} c d=(\mathrm{Mo} / \mathrm{Mt}) \mathrm{X} 100$ donde; Mo es el numero de campos completos en el metadato del MUJ y Mt es el total de campos sugeridos y obligatorios por el estándar utilizado (9). Esto con el fin de establecer que tan documentado esta el metadato de corales del MUJ en aras de su divulgación (9). El criterio mínimo utilizado para definir si el metadato es lo suficiente-mente confiable para ser liberado al público y fue de un $70 \%$. La pauta del 
porcentaje de este índice fue arbitraria, asumiendo que por debajo del 70\% la documentación de los registros biológicos es inadecuada para interoperar con otros metadatos del mismo grupo y para ser publicado.

\section{Resultados}

El metadato documentó un inventario resumido y actualizado de la información existente al interior de la base de datos de la colección de escleractinios del MUJ tales como: sitio donde reposa la colección: $\mathrm{Mu}-$ seo Javeriano, colección de invertebrados marinos; sitios donde se colectó: ARCO, MAG, SAN, TAY, MOR; requerimientos para realizar un proceso personalizado de pedido (préstamos); progreso de la colección y las condiciones de uso y acceso entre otras.

La cantidad del contenido de la información para documentar el metadato fue de un $76.6 \%$, valor arrojado por el índice de metadatos y correspondiente a la cantidad de campos llenos, al compararlo contra el total de información que debe estar completa en el estándar utilizado (9).

Finalmente, este metadato suministró un perfil de características sobre el estado de completitud, calidad de completo de la información inherente a los ejemplares de la colección de corales hermatípicos del MUJ; arrojó información de la integridad taxonómica a nivel de género y a su vez mostró información sobre los requerimientos del usuario con la institución en caso de necesitarse apoyo del material referenciado (Tabla 1).

\section{Discusión}

El metadato en ciencias cobra un valor agregado para las colecciones biológicas, por que organiza, clasifica, estandariza, y describe la información de manera general y por rangos para cualquier grupo biológico o conjunto de datos (2). Sin embargo, pese a que existe esta implementación del metadato en Colombia, sugerida por el IAvH, para las colecciones biológicas (14), los curadores del MUJ y de otras ins- tituciones no liberan la información que contienen al interior de sus bases de datos. Esto es debido a la ambigüedad que existe con respecto al reconocimiento de los derechos de autor (el que colecta, determina, clasifica, o genera el metadato).

Para solucionar este problema es importante incentivar a los curadores y científicos a publicar la información existente en sus colecciones (5) y a que las revistas nacionales dentro de sus lineamentos permitan la publicación de metadatos. Una vez la información haya sido documentada y publicada, esta podría estar disponible en red. De acuerdo con Rivera (9), simultáneamente se podría ofrecer atención adecuada y oportuna a los usuarios de la información, quienes requieren acceso fácil y rápido a la misma, con poco o ningún costo, para así difundirla a un mayor público y a la comunidad científica nacional e internacional (14).

De otro lado, el índice de intercambio de información aplicado al metadato de la colección de corales hermatípicos del MUJ indica que este se encuentra cerca al valor definido como "límite", lo que indicaría la mínima documentación requerida no solo para realizar el metadato, sino también para que al publicarlo su información sea útil (5). No obstante, el indicador de intercambio, índice de cantidad, posee limitaciones en cuanto a la cantidad de información que se debe tener en cuenta para obtener resultados robustos (13), debido a que esta cantidad depende de: (i) la información disponible de los registros biológicos en cada colección; (ii) la manera de ingresar la información a las bases de datos; (iii) la forma de registrar datos en la libreta de campo (iv) completar información post-colecta. Por esta razón es importante tener en cuenta una plataforma específica para documentar metadatos, ej. Dublín Core, ya que de los campos específicos recomendados a diligenciar, se adquieren los items para documentar la información de cada colección de acuerdo a los requerimientos de cada institución. En este caso los ítems fueron arbitrarios y además se estableció un estándar especifico de con- 
Tabla 1. Metadato de Corales Escleractinios del Caribe colombiano referenciados en el Museo de Historia Natural Lorenzo Uribe S.J. Los campos en negrilla son obligatorios (mínimos oligatorios). Estándar para la documentación de conjunto de datos.

\section{CITACION}

Cítese: Martha Yaneth Vallejo.2005. Metadato de corales hermatípicos del Caribe colombiano: Colección de Invertebrados (Cnidaria: Anthozoa: Scleractinia) Museo de Historia Natural Lorenzo Uribe S.J. - Pontificia Universidad Javeriana.

\begin{tabular}{|c|c|}
\hline \multicolumn{2}{|l|}{ INFORMACIÓN DE CITACIÓN } \\
\hline AUTOR & Martha Yaneth Vallejo \\
\hline FECHA & 20050625 \\
\hline TITULO DEL DOCUMENTO & $\begin{array}{l}\text { Metadato de corales hermatípicos del Caribe colombiano, Museo } \\
\text { de Historia Natural Lorenzo Uribe S.J., Colombia }\end{array}$ \\
\hline EDITOR & Universidad Colegio Mayor de Cundinamarca \\
\hline PUBLICADOR & NOVA \\
\hline EDITORIAL & Imprenta Nacional de Colombia \\
\hline LUGAR DE PUBLICACIÓN & Bogotá, Colombia \\
\hline TIPO DE CITACIÓN & Colección de referencia \\
\hline EDICIÓN O VERSIÓN & Base de datos en Excel - XP. \\
\hline VOLUMEN & 3 \\
\hline \multicolumn{2}{|l|}{ SERIE } \\
\hline NUMERO & 4 \\
\hline PAGINAS & 8 unds (44 a 51) \\
\hline HIPERVÍNCULO & www.revistanova@unicolmayor.edu.co \\
\hline FECHA DE CONSULTA & 20050927 \\
\hline OTROS DETALLES & $\begin{array}{l}\text { El metadato se encuentra en CD ROM y en publicación disponible al } \\
\text { público en la Pontificia Universidad Javeriana. }\end{array}$ \\
\hline DEPOSITORIO DE LA CITACIÓN & laacosta@javeriana.edu.co \\
\hline PROPOSITO & $\begin{array}{l}\text { Para información al público en general y para facilitar la investigación } \\
\text { y el conocimiento sobre la biodiversidad a cualquier nivel (Familia- } \\
\text { género y especie) dentro de la colección. }\end{array}$ \\
\hline \multicolumn{2}{|l|}{ COBERTURA GEOGRÁFICA (c) } \\
\hline $\begin{array}{l}\text { DESCRIPCIÓN DE LA EXTENSIÓN } \\
\text { GEOGRÁFICA }\end{array}$ & $\begin{array}{l}\text { Las muestras se colectaron en el Caribe colombiano en las } \\
\text { ecorregiones de ARCO, MAG, SAN, TAY, MOR. }\end{array}$ \\
\hline \multicolumn{2}{|l|}{ RANGO DE ALTITUD (C) } \\
\hline ALTITUD MÍNIMA & 0 \\
\hline ALTITUD MÁXIMA & 30 \\
\hline UNIDADES DE ALTITUD & Metros \\
\hline PALABRAS CLAVE & Rango, Batimétrico \\
\hline \multicolumn{2}{|l|}{ TEMA ( C) } \\
\hline TESAURO DE TEMAS & lavH \\
\hline PALABRAS CLAVE DE TEMA & Base de datos, Corales, Taxónomia. \\
\hline $\begin{array}{l}\text { LUGAR ( C) } \\
\text { RECURSO DE AUTORIDAD DE }\end{array}$ & Colombia, Caribe colombiano. \\
\hline LUGARES & GACETERO DANE, SIM INVEMAR. \\
\hline PALABRAS CLAVES DEL LUGAR & Departamentos, Ecorregiones. \\
\hline $\begin{array}{l}\text { PALABRAS CLAVE DE TIEMPO (C) } \\
\text { RECURSO DE AUTORIDAD DE }\end{array}$ & \\
\hline PALABRAS CLAVE DE TIEMPO & GACETERO DANE \\
\hline PALABRAS CLAVE & Fecha \\
\hline
\end{tabular}




\begin{tabular}{|c|c|}
\hline PALABRAS CLAVES (C) & Cnidaria: Anthozoa: Scleractinia. \\
\hline \multicolumn{2}{|l|}{ RECURSO DE AUTORIDAD } \\
\hline $\begin{array}{l}\text { PALABRAS CLAVE } \\
\text { SISTEMA TAXONÓMICO (C) }\end{array}$ & SISTEMA TAXONÓMICO (C) \\
\hline $\begin{array}{l}\text { SISTEMA DE CLASIFICACIÓN O } \\
\text { AUTORIDAD (C) }\end{array}$ & Cairns (1999); Veron(2000) \\
\hline \multicolumn{2}{|l|}{ REFERENCIA } \\
\hline $\begin{array}{l}\text { RESPONSABLE DE LA } \\
\text { IDENTIFICACIÓN(C) }\end{array}$ & Acosta Alberto. Ver contacto anexo 1. \\
\hline \multicolumn{2}{|l|}{ INFORMACIÓN DEL CONTACTO 1} \\
\hline ORGANIZACIÓN A CONTACTAR & PONTIFICIA UNIVERSIDAD JAVERIANA ( MPUJ) \\
\hline PERSONA DE CONTACTO & Acosta Alberto. \\
\hline CARGO & Curador de colección de corales y zoanthideos \\
\hline DIRECCIÓN DE CONTACTO & Carrera $7 \mathrm{~N}^{\circ} 43-82$ \\
\hline CIUDAD O MUNICIPIO & BOG \\
\hline SUBDIVISIÓN ADMINISTRATIVA & Cundinamarca \\
\hline PAís & $\mathrm{CO}$ \\
\hline TELÉFONO DE CONTACTO & (57-1) 3208320 Ext. 4082 \\
\hline $\begin{array}{l}\text { FAX DE CONTACTO } \\
\text { DIRECCIÓN DE CORREO }\end{array}$ & (57-1) 3208320 Ext. 4128 \\
\hline ELECTRÓNICO & laacosta@javeriana.edu.co \\
\hline \multicolumn{2}{|l|}{ HORARIO } \\
\hline HORA INICIAL & $090000-500$ \\
\hline HORA FINAL & $170000-500$ \\
\hline INSTRUCCIONES DE CONTACTO & Correo Electrónico o vía telefónica \\
\hline $\begin{array}{l}\text { PROCEDIMIENTO TAXONÓMICO } \\
\text { INTEGRIDAD TAXONÓMICA }\end{array}$ & Método \\
\hline GÉNEROS TOTALES & $\begin{array}{l}19 \text { GENEROS, 11FAMILIAS, 30ESPECIES } \\
\text { Agaricia-Cladocora-Colpophyllia-Dichocoenia-Diploria-Eusmilia- } \\
\text { Favia-Leptoseris-Isophyllastrea-Madracis-Manicina-Meandrina- } \\
\text { Montastrea- Mussa- Mycetophyllia Porites- Scolymia- Siderastrea- } \\
\text { Tubastrea. }\end{array}$ \\
\hline TIPO DE EVIDENCIA & Ejemplares en seco (esqueletos de juveniles 2-4cm de diámetro). \\
\hline REPOSITORIO (C): & $\begin{array}{l}\text { Pontificia Universidad Javeriana, Museo de Historia Natural. Lorenzo } \\
\text { Uribe S.J. Contacto } 2 .\end{array}$ \\
\hline \multicolumn{2}{|l|}{ TAXONÓMICA } \\
\hline \multicolumn{2}{|l|}{$\begin{array}{l}\text { CLASIFICACIÓN TAXONÓMICA (C) } \\
\text { NOMBRE DE LA CATEGORIA }\end{array}$} \\
\hline VALOR DE LA CATEGORÍA & Cnidaria \\
\hline NOMBRE COMÚN & Corales \\
\hline CONDICIONES DE ACCESO & $\begin{array}{l}\text { Solicitar permiso al curador de la Colección de corales y Zoanthideos } \\
\text { para tener acceso a la base de datos de la colección o en su defecto } \\
\text { al repositorio. }\end{array}$ \\
\hline CONDICIONES DE USO & $\begin{array}{l}\text { El uso es restringido dependiendo del tipo de investigador y } \\
\text { proyecto para el cual se va a usar. La Universidad y el curador se } \\
\text { reservan el derecho de admisión a la base de datos. }\end{array}$ \\
\hline \multicolumn{2}{|l|}{ CONTACTO } \\
\hline \multicolumn{2}{|l|}{ INFORMACIÓN DEL CONTACTO 2} \\
\hline ORGANIZACIÓN A CONTACTAR & PONTIFICIA UNIVERSIDAD JAVERIANA (MPUJ) \\
\hline PERSONA DE CONTACTO & Alberto Acosta \\
\hline CARGO & Profesor de Biología. PUJ. \\
\hline
\end{tabular}




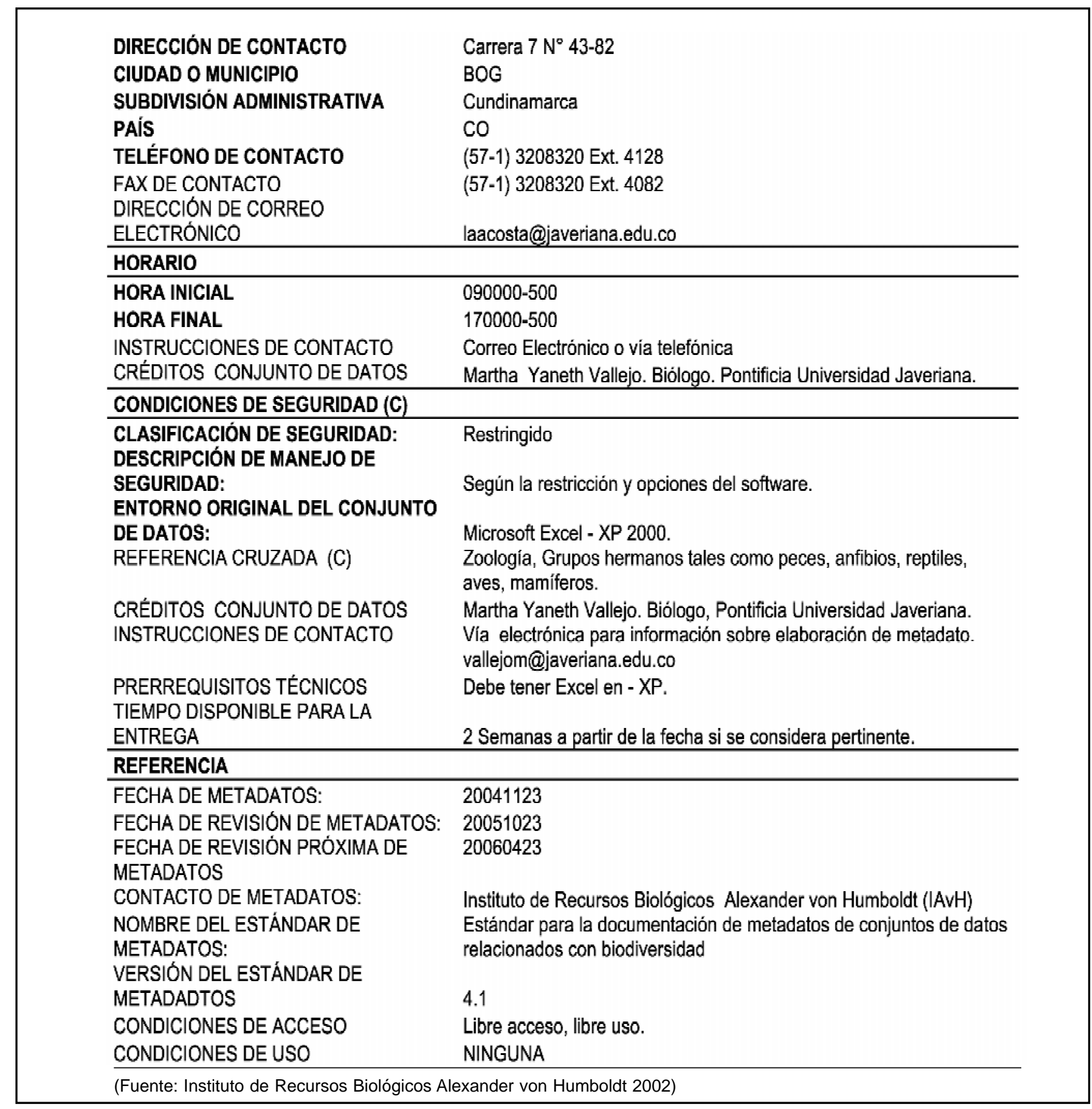

junto de datos (5). No obstante, la documentación del metadato de la colección biológica del MUJ, logro minimizar datos y maximizar información (12). Dicha información evita como lo documenta Rivera (9), duplicar esfuerzos en la búsqueda de otras bases de datos del mismo grupo biológico con igual información o por el contrario, que en conjunto tengan información complementaria de manera que puedan ser útiles para los investigadores (14).
En este contexto, el metadato de la colección del MUJ es complementario con otros que manejen el mismo grupo biológico en Colombia, como es el caso de Invemar, pues presenta información donde los componentes faltantes, casillas por diligenciar, en el metadato de una colección están en otra; ya sea por poseer atributos diferentes o por que la complementación de datos a posteriori fue eficiente; ej: ver metadato del MHNMC en SIM o web del IAvH. 
Es decir, los metadatos de las colecciones de un mismo grupo biológico, sumados proporcionan sino la totalidad, por lo menos parte de la información como en este caso, de los datos anexos a los corales hermatípicos de referencia, escleractinios del Caribe colombiano. Por ello y conforme con Rivera-Gutiérrez (9), el generar información de un metadato, “compromete" a los curadores o gestores del mismo, a actualizar en forma periódica el estándar adoptado, compararlo con las diferentes normas internacionales y requerimientos institucionales, asegurándose que estos cumplan el mínimo de datos y campos diligenciados para facilitar la integración de metadatos de diversos grupos y organizaciones.

Es importante recalcar que entre mas completa y veraz la información de la base de datos y el metadato de las colecciones de los museos (14), mayor confiabilidad se tendrá al momento de hacer sus consultas y de decidir sobre cuales variables puede profundizar cada investigador (13).

Cabe mencionar que la implementación y publicación de este metadato, es un aporte en el ámbito de la divulgación de un recurso biológico como lo son las colecciones, donde el modelo ha sido validado y ha producido una serie de publicaciones internacionales de divulgación científica que avalan su utilidad (3), y donde la información se basa como lo indica Rivera (9), en la veracidad de la información ingresada de mano con los investigadores y taxónomos expertos.

Por último, se reconoce que se necesitarán estándares específicos de metadatos para otros temas biológicos, ej: áreas protegidas y flora neotropical, importantes para conocer y diseñar planes de conservación. Para muchos de estos temas, no hay estándares predominantes o emergentes (4), que a través de procesos lleguen a un consenso que implique la participación de los principales actores dentro del tema de interés. El primer paso en este proceso es hacer que los museos identifiquen la prioridad de los temas relevantes y los participantes pertinentes.

\section{Agradecimientos}

A Doris González, al profesor Camilo Andrade y los alumnos del curso invertebrados (2004) por la colaboración en la identificación de escleractinios (MUJ) y digitación preeliminar de la base de datos. Este trabajo fue realizado dentro del proyecto Organización y sistematización de las colecciones algales y de invertebrados no artrópodos de las colecciones biológicas del Museo Javeriano financiado por Vicerrectoría de la Pontificia Universidad Javeriana (PUJ) Código 1583/ 2004.

\section{Referencias}

1. Lazinger S. Digital Preservation and Metadata. Englewood, Colorado: Libraries Unlimited; 2001. 256 p.

2. Weibel S. The Metadata Landscape: Convetions for Semantics-Syntax and Structure in the Internet Commons. Resúmenes Evento Metadiversity II: Nfais. en: http/ www.nfais.org/Metadiversityl.asp.

3. Enric PO. Interoperabilidad de Metadatos en Sistemas Distribuidos. Tesis Doctoral, Universitat Pompeu Fabra; 2003. 92 p. 4. Llorente J, Luna, compiladores. Museos y la Conservación de la Biodiversidad, Taxonomía Biológica. UNAM, Fondo de Cultura Económica. México: Ediciones Científicas Universitarias; 1994. 325 p.

5. Llorente J, Luna, compiladores. Bases de datos en Taxonomía y Colecciones Científicas, Taxonomía Biológica. UNAM, Fondo de Cultura Económica. México: Ediciones Científicas Universitarias; 1994. p. 148.

6. Rivera HF, Suárez AM, Bello JC. Estándar de Información para Registros Biológicos. Sistema de Información sobre Biodiversidad. Instituto Alexander Von Humboldt, Bogotá D.C. Colombia; 2002. p: 30-56.

7. Instituto de Investigaciones y Recursos Biológicos Alexander Von Humboldt (IAvH). Sistema de información en biodiversidad y Sistemas de Indicadores de Seguimiento de Política de Biodiversidad. en: http://www.humboldt.org.co/sib

8. Dublin C. History of the Dublin Core Metadata Initiative; 2002. en: http://dublincore.org/about/history

9. Rivera H, Suárez A, Bello J. Estándar para la Documentación de Metadatos de Conjuntos de Datos relacionados con Biodiversidad, IAvH. Villa de Leiva Colombia, 2003; 66 p.

10. National Biological Service. Draft Content Standard for National Biological Information Infrastructure Metadata. Denver: Colorado 1995; en: http://www.nbii.gov/factsheet/ factsheet 3.html.

11. Federal Geographic Data Committee (FGDC). National Biological Information Infraestructure, Content Standard for Digital Geospatial Metadata. Washington D.C.: USA. 2000.

12. FGDC. National Biological Information Infraestructure, Contect Standard for Digital Geospatial Metadata, Biological Data Profile Workbook. Washington D.C.: USA. 2001; 1450 p.

13. Vivas AJ. Evaluación de la Información de Colecciones Biológicas sobre los Géneros Elaeagia y Faramea (Familia Rubiaceae) en la Región Andina Colombiana. Trabajo de Grado, programa de Biología Facultad de Ciencias, Universidad de Popayán Colombia. 2000; 67 p. Versión Electrónica 2.0: IavH; 2002; en: www.humboldt.org.co/sib

14. Rivera HF. Documento de trabajo. Fundamentos para la implementación de un Catálogo de Metadatos de conjuntos de datos relacionados con Biodiversidad. Bogotá; 2003. 34 p. 